\title{
CUERPOS ACADÉMICOS EN ESCUELAS NORMALES: LA COLEGIALIDAD PARA LA PRODUCCIÓN Y DIFUSIÓN DEL CONOCIMIENTO
}

\section{ACADEMIC GROUPS IN NORMAL SCHOOLS: COLLEGIALITY FOR THE PRODUCTION AND DISSEMINATION OF KNOWLEDGE}

doi: 10.21555/rpp.v33i33.2410

\section{María Guadalupe Loza Jiménez}

maríaguadalupe.loza@upaep.edu.mx

https:// orcid.org/0000-0003-4106-5233
Martha Leticia Gaeta González

marthaleticia.gaeta@upaep.mx

https:/ / orcid.org/0000-0003-1710-217X

Escuela Normal «Instituto Jaime Torres Bodet», Universidad Popular Autónoma del Estado de Puebla

Recibido: octubre 12, 2021 - Aceptado: octubre 26, 2021

\section{RESUMEN}

Las Escuelas Normales son instituciones de educación superior, responsables de la formación de docentes de educación básica en México. Desde 2009, han respondido a una política pública establecida para la conformación de Cuerpos Académicos. Estos grupos están integrados por profesores de tiempo completo con intereses comunes en temas disciplinares o multidisciplinares, con la finalidad de realizar investigaciones colegiadas para generar y aplicar conocimiento. La presente investigación tiene como objetivo analizar la contribución de la colegialidad para la producción y difusión del conocimiento en los Cuerpos Académicos de las Escuelas Normales del estado de Puebla. Con un enfoque cualitativo-interpretativo, se entrevistó a cinco docentes de Cuerpos Académicos de tres Escuelas Normales, considerando como categorías de análisis: colegialidad, producción y difusión del conocimiento. Los hallazgos permiten identificar algunas características de la cultura colegiada (prácticas y estrategias), que inciden en la producción y difusión del conocimiento que se genera al interior de los Cuerpos Académicos de las Escuelas Normales del Estado de Puebla.

Palabras clave: cooperación en investigación, conocimiento científico, difusión del saber científico, grupos académicos, formación de docentes. 


\begin{abstract}
«Normal Schools» are higher education institutions responsible for the training of elementary school teachers in Mexico. Since 2009 they have responded to a public policy established for the formation of Academic groups. These groups are made up of full-time professors with common interests in disciplinary or multidisciplinary subjects to carry out collegiate research to generate and apply knowledge. The present study aims to analyze the contribution of collegiality for the production and dissemination of knowledge in Normal Schools' Academic groups in the state of Puebla. With a qualitative-interpretive approach, five teachers from Academic groups from three Normal Schools were interviewed, considering collegiality, production, and dissemination of knowledge as analyzing categories. The findings show some characteristics of the collegiate culture (practices and strategies) that affect the knowledge generated within the Academic groups of the Normal Schools of the State of Puebla.
\end{abstract}

Keywords: Research cooperation, Scientific Knowledge, Dissemination of Scientific Knowledge, Academic Groups, Teacher Training.

\title{
1. INTRODUCCIÓN
}

Desde 1996, surge en México, el Programa de Mejoramiento del Profesorado (PROMEP), cuyo objetivo es contribuir a que los Profesores de Tiempo Completo (PTC) de las instituciones públicas de Educación Superior, alcancen las capacidades para realizar investigación y docencia, se profesionalicen, articulen y consoliden en grupos de pares llamados Cuerpos Académicos (CA). Pero es hasta 2009 que las Escuelas Normales (EN) son convocadas para participar en dicho programa que, a partir de 2012, se denomina Programa para el Desarrollo Profesional Docente (PRODEP) y define a los CA como (DOF, 2013):

Grupos de profesores / as de tiempo completo que comparten una o varias líneas de generación de conocimiento, investigación aplicada o desarrollo tecnológico e innovación en temas disciplinares o multidisciplinares, y un conjunto de objetivos y metas académicas, [...] situación que les permite erigirse como las células de la academia y representar a las masas críticas en las diferentes áreas del conocimiento que regulan la vida académica de las Instituciones de Educación Superior (p. 11).

El primer CA en las EN del estado de Puebla, surgió en 2011. Al momento de la presente investigación, existen 21 CA (GobMx, 2021). Las características de los CA varían dependiendo de su grado de consolidación; en formación (CAEF), en consolidación (CAEC) o consolidado (CAC), a partir de la solidez y madurez de las Líneas de Generación y Aplicación Innovadora del Conocimiento (LGAC) que cultivan, por el diseño conjunto de proyectos innovadores, los productos académicos que generan, el grado profesional de los integrantes, el reconocimiento al perfil deseable y la forma de trabajo colegiado, que implica la integración de redes de colaboración o cooperación, y movilidad académica (DOF, 2013). Las LGAC son definidas por el PRODEP como: «Una serie coherente de proyectos, actividades o estímulos que profundizan en el conocimiento como producto de la investigación básica y aplicada, con un conjunto de objetivos y metas de carácter académico, en temas disciplinares o multidisciplinares» (DOF, 2013, p. 18).

Del total de CA en la entidad, tres son CAEC y el resto son CAEF, sin que exista algún CAC (GobMx, 2021). Entre los CA de las EN poblanas se cultivan 25 LGAC, orientadas al ámbito educativo con énfasis en la formación de docentes. Con base en la clasificación temática del Consejo Mexicano de Investigación Educativa (COMIE), las LGAC involucran: 
procesos de formación (13); estudiantes, maestros y académicos en investigación educativa (4); aprendizaje y desarrollo (3); filosofía, teoría y campo de la educación (2); conocimientos disciplinares en México (2), y entornos virtuales de aprendizaje (1) (GobMx, 2021).

Generalmente, las investigaciones en dichos CA surgen de las problemáticas contextuales y producen conocimiento en el campo educativo, y resulta importante que, de manera colegiada, la producción se difunda y se ponga a disposición de los usuarios. Sin embargo, en el estado de Puebla se desconoce el funcionamiento de los CA que se han conformado en las EN. Si bien, al interior de las instituciones se está al tanto de la forma en que se realizan algunos procesos, se ignora acerca de los modos de producción y difusión del conocimiento. Es aquí donde surgen algunas preguntas que orientan la presente investigación. De manera general: ¿cómo contribuye la colegialidad para la producción y la difusión del conocimiento en los Cuerpos Académicos de las Escuelas Normales Públicas del estado de Puebla? Y, específicamente, ¿qué aspectos de la colegialidad inciden para la producción y difusión del conocimiento en los CA?, ¿qué estrategias definen los CA para la producción y difusión del conocimiento que generan?

Con base en estos cuestionamientos, el objetivo general de la presente investigación es analizar la contribución de la colegialidad para la producción y difusión del conocimiento en los Cuerpos Académicos de las Escuelas Normales Públicas del estado de Puebla, México.

Conocer las características de la colegialidad en los CA al interior de las EN del estado de Puebla, y su relación con la producción y difusión del conocimiento que en ellos se genera, puede justificarse por varias razones: 1) se trata de un tema poco explorado; 2) la colegialidad es un rasgo imprescindible en la investigación educativa actual y su análisis resulta necesario; 3) es ineludible un acercamiento a los procesos académicos en relación con la producción y difusión del conocimiento en las instituciones que forman a los docentes de México, y 4) recoger la experiencia emanada para la producción y difusión del conocimiento en los CA, quizá pueda replicarse en otras EN o repensarse en correspondencia con sí misma.

\section{MARCO REFERENCIAL}

Los CA se conforman por un grupo de personas que interactúan con base en un propósito común, que comparten saberes y se retroalimentan para el logro de un mismo beneficio, por lo que resulta interesante determinar las características para la producción y difusión del conocimiento, a partir de un enfoque definido como estudio de la vida de los grupos humanos y del comportamiento del hombre. Se trata del interaccionismo simbólico (IS), término introducido por Blumer, en 1937, con una importante influencia teórica en trabajos de corte cualitativo en el campo de la antropología, la psicología social y la sociología política.

Las tres premisas del IS, según Blumer (1982, p. 2), son:

1. Los seres humanos actúan hacia diversas cosas sobre las bases de los significados que esas cosas tienen para ellos.

2. El significado de tales cosas emerge de la interacción social que uno tiene con los asociados a uno.

3. Los significados dependen y se modifican a través de un proceso interpretativo usado por la persona en su trato con las cosas que encuentra. 
Los principios básicos del IS, se pueden identificar en el quehacer cotidiano de los CA, debido a que la capacidad de pensamiento de los integrantes se modela por la interacción social que practican, a través de la cual aprenden significados que les permiten actuar e interactuar de cierta manera. La integración de un grupo epistémico se encuentra en relación con lo que significa para los docentes realizar investigación educativa y participar en procesos académicos; ello se confirma, altera o modifica cuando interactúan desde la práctica de un trabajo colegiado y pueden -o no- otorgarle un significado diferente, dependiendo de su capacidad para interactuar consigo mismos. Esto, a su vez, les permite examinar los posibles cursos de acción, y valorar sus ventajas y desventajas relativas para luego elegir uno. Cada integrante manifiesta significados al resto del grupo y, de esta manera, se ve influenciado por las actitudes y significados de los otros pares logrando, como lo expresa Blumer (1982, p. 12), «que las muestras de comportamiento comunitario hagan que sus líneas de acción encajen o se adopten recíprocamente».

Para la comprensión del desarrollo de los colectivos de trabajo es de suma importancia el papel de la interacción ya que, como lo menciona Rizo (2006), es un proceso en el que una pluralidad de acciones se relaciona recíprocamente y, en este sentido, estudia procesos interpersonales, formando parte de grupos, y no acciones aisladas. El autor considera que "gran parte de las investigaciones en psicología social se sitúan a nivel de la interacción, y de esta surge el interés por aspectos como la cohesión, el liderazgo, la dinámica de grupos y la comunicación» (p. 54).

\subsection{Colegialidad}

El término colegialidad «tradicionalmente se ha utilizado para identificar la calidad de la unión entre las personas que trabajan de forma coordinada y colaborativa, con un fin u objetivo común» (Bakieva, 2011, p. 8), que les permite aprender unos de otros y alcanzar un desarrollo mutuo. Según Martínez (2015), tiene que ver con intereses y metas en común, que propicien discusiones para reflexionar de manera formal o informal, y señala que «en la colegialidad se establece una relación de horizontalidad [...] es decir, se discute y se trabaja entre pares» (p. 7).

Partiendo de estas aportaciones y para fines de la presente investigación, podemos señalar que colegialidad es: una condición entre pares en la que se establecen vínculos personales y profesionales, con base en significados comunes y un mismo interés en un área o disciplina. Su finalidad es impulsar el cumplimiento de objetivos y metas, a través de un trabajo continuo, compartido, comprometido y ético, cuya base es la reflexión, la crítica, el intercambio de percepciones y experiencias, para favorecer la investigación y/o la innovación educativa.

Esta condición puede ser vista como un sistema al que podemos llamar sistema colegiado, ya que en él existen normas y procedimientos que regulan el funcionamiento o comportamiento de los integrantes del grupo o colectividad; lo mismo que a las actividades que realizan en conjunto para contribuir a los propósitos comunes, podemos definirlo como trabajo colegiado; este se concibe por los profesores como algo más que la pertenencia a un grupo de trabajo; más bien refieren un «grupo de compañeros que les permite aprender, en un ambiente de cuestionamiento en el que se gesta un desarrollo académico y una condición de posibilidad en su profesionalidad» (Rayas, 2011, p. 8). Esta forma de trabajo -que implica ciertas características de cotidianidad académica- es a lo que Becher (1999) llama cultura colegiada.

Los principales elementos del trabajo colegiado en los CA de las EN, están en relación con: a) el trabajo colaborativo; b) el trabajo en equipo, y c) el trabajo en redes académicas, cuya adecuada funcionalidad favorece una verdadera colegialidad. 


\section{Figura 1}

Esquema conceptual del trabajo colegiado en Cuerpos Académicos, y su relación con la producción y difusión del conocimiento

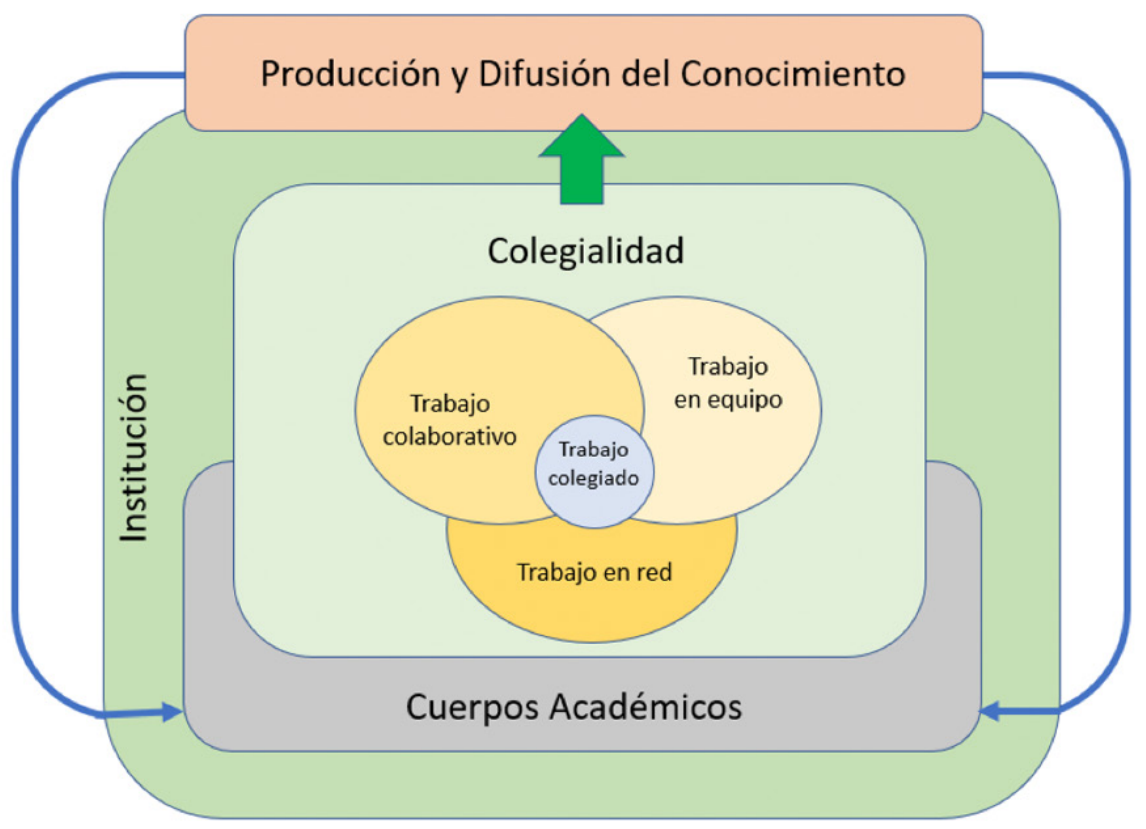

a. La colaboración se ha definido como «un enlace formal de dos o más participantes autónomos, cuya relación no es de tipo contractual, y tiene como objetivo el logro de metas sustantivas o simbólicas que cada uno de los participantes no podría lograr independientemente» (Domínguez-Gaona et al., 2015, p. 123).

Para Antúnez (1999), la colaboración entre docentes es un modo de trabajar en que se comparten recursos para alcanzar unos propósitos específicos, durante un periodo de tiempo determinado. Es voluntario, en términos de igualdad entre pares; se basa en la lealtad y confianza recíproca; implica un planteamiento ideológico, y supone «el diseño de lo que se pretende alcanzar o desarrollar, [al] acordar la metodología de trabajo y discutir y evaluar en común los resultados» (p. 95).

La actividad de investigación que se realiza en los CA y su vinculación con el trabajo colaborativo, propician el concepto de investigación colaborativa, que de acuerdo con Cano (2010), «es un valioso enfoque para construir el conocimiento entre y por los profesores e investigadores» (p. 61).

b. El trabajo en equipo, de acuerdo con Morelli (2000), se conforma por «un grupo de personas que se reúnen para trabajar de manera coordinada en la ejecución de un proyecto. Por la teoría sistémica, el equipo responde al resultado final y no a cada uno de sus miembros de manera independiente» (p. 1).

El trabajo en equipo es una característica de la forma de organización de los CA a la que hacen referencia las políticas públicas del PRODEP, ya que como menciona Antúnez (1999), el trabajo en equipo dentro del ámbito docente: 
[...] se justifica como un mecanismo para proporcionar una atmósfera que anime a los integrantes a trabajar con entusiasmo, o para aumentar el autoconcepto y la autoestima del profesorado y su sentimiento de propiedad y de pertenencia respecto al centro escolar [...] es uno de los factores clave relacionados con la mejora de los aprendizajes de los alumnos (p. 95).

El trabajo en equipo que los docentes deben realizar al interior de los CA no se separa de la funcionalidad que se le otorga al mismo, como lo refiere Morelli (2000), tiene que ver con las cinco «C»: 1) complementariedad; 2) coordinación; 3) comunicación; 4) confianza, y 5) compromiso. A esta propuesta, Espinosa (2008) agrega otra «C», que corresponde a colaboración.

c. El trabajo en redes es otra de las características de las comunidades epistémicas y en general de los procesos de producción del conocimiento. Las redes de acuerdo con López, (2010), constituyen un instrumento de análisis y trabajo en la sociedad, y tienen como propósito (ANUIES, 2019):

[...] el estudio de temas disciplinares o multidisciplinares cuya relevancia amerite un esfuerzo colectivo de alcance regional o nacional, sistemático y permanente. Su labor se sustenta en la colaboración, la coparticipación, la corresponsabilidad y en el intercambio continuo de información con base en la experiencia y competencias de sus miembros (p. 1).

Algunas de las ventajas del trabajo en red para el desarrollo profesional docente, son las de combatir el aislamiento del profesorado ofreciendo apoyo social, y estimular la articulación y explicitación del conocimiento tácito acerca del aprendizaje y la enseñanza (López, 2008). Al participar en redes, los integrantes de los CA propician una investigación dentro de comunidades que comparten supuestos conceptuales y metodológicos, basados principalmente en aspectos de formación docente.

\subsection{Producción del conocimiento}

La producción del conocimiento consiste en lograr aportaciones a un tema, disciplina, campo determinado, resultado de un proceso de investigación en el que se involucran diversos actores y sus prácticas, cuya finalidad es la resolución de problemas sociales.

Tradicionalmente, el conocimiento se ha producido de una forma delimitada, gracias al contexto disciplinar que lo produce, legitima y valora, y al que Gibbons et al. (1997) han llamado modo 1. Se presenta como un conocimiento homogéneo, que se genera en la institución (universidad), atendiendo a una problemática disciplinar, que es jerárquico y se difunde en el gremio específico de la disciplina, y es valorado por los pares.

Gibbons et al. (1997) también proponen el modo 2, en el que se consideran diferentes lugares potenciales para generar conocimiento: el contexto va más allá de las instituciones y considera a la sociedad, la industria y las empresas. Quienes practican la ciencia son diferentes sujetos del contexto, que con diversas metodologías y abarcando problemáticas de manera transdisciplinar o multisectorial, organizan el conocimiento de manera no jerárquica, a través de diversas estructuras. Debido a esa condición transdisciplinaria se incrementa la difusión, y se avanza a nuevos contextos de aplicación y uso (Sañudo, 2010).

Carayannis y Campbell (2012, referidos por Chíquiza, 2015) proponen un nuevo modo de la producción del conocimiento al que llaman modo 3, el cual promueve el enfoque democrático de la innovación -surgido de la preocupación de la crisis ecológica del planeta-, 
bajo un ámbito político, económico y epistemológico. Este modo de producir conocimiento se sustenta en el desarrollo sostenible y vincula la innovación, el espíritu emprendedor y la democracia. Se fundamenta en el enfoque de sistemas para la creación de conocimientos, difusión y utilización; las redes de innovación; agrupaciones de conocimientos, y modelos de investigación fractal de la educación y ecosistema de innovación.

Los tres modos presentan aspectos comunes, pero cada uno posee sus respectivas peculiaridades; surgen en distintos momentos históricos, bajo condiciones epistémico-políticas diferentes, con intereses y preguntas de investigación que provienen de contextos diversos, con fundamentación y relación con la comunidad disímiles, y con formas de trabajo que difieren entre sí.

\subsection{Difusión del conocimiento}

La comunicación de la ciencia incluye una serie de términos que hacen referencia a distintos procesos: difusión, divulgación o diseminación, son conceptos que frecuentemente se enlazan con términos como: alfabetización científica, cultura científica o comprensión pública de la ciencia.

Pascuali (1990, referido por Sañudo, 2014, p. 31), realiza una distinción entre los principales conceptos vinculados con la comunicación de la ciencia, proponiendo las siguientes definiciones:

- Difusión: Es la transmisión de información científica por parte de expertos a audiencias generalmente educadas o instruidas que, aunque no son expertas en el tema, pueden entender el mensaje debido a que en este se utiliza un lenguaje menos especializado y más accesible.

- Divulgación: Es el envío de mensajes, elaborados mediante la transcodificación de lenguajes crípticos a lenguajes comprensibles, a la totalidad del universo perceptor disponible.

- Diseminación: Es la remisión de mensajes, elaborados en lenguajes especializados, a perceptores selectivos y restringidos. En esta acción, el flujo de información se da entre los científicos y expertos, quienes comprenden el lenguaje especializado.

De acuerdo con Sañudo (2010), la difusión es el rubro más conocido y relevante del proceso de distribución del conocimiento, que se realiza primordialmente por medio de revistas y artículos científicos. Esta difusión sirve de vehículo entre los resultados de la investigación y los colegas expertos e interesados en el área que, tal como menciona Hernández (2001), puede generarse de dos maneras: formal e informal. La primera alude a la comunicación de la producción mediante su publicación, y la segunda consiste en la participación en eventos académicos o por contactos personales (colegios invisibles).

Los investigadores integrantes de un CA buscan difundir, divulgar o diseminar los resultados de sus investigaciones. Incluso, existe una idea generalizada entre los miembros de estos grupos epistémicos: «Investigación que no se publica... ¡no existe!». De tal forma que ellos pueden participar en foros, mesas de discusión con expertos, congresos, mesas redondas, e incluso escribir artículos para revistas especializadas, capítulos de libros, libros o periódicos o revistas de consulta popular. 


\section{METODOLOGÍA}

La presente investigación adopta un enfoque cualitativo-interpretativo. Los métodos, técnicas, estrategias e instrumentos se encuentran, en este caso, en la lógica de observar necesariamente algún aspecto de la realidad que viven los CA de las Escuelas Normales del Estado de Puebla, en relación con las características de la colegialidad para la producción y difusión del conocimiento.

Del total de CA, dos cuentan con mayor antigüedad, dos con una antigüedad media y uno con registro reciente; dos de ellos son reconocidos por el PRODEP como en consolidación y tres en formación. Sus LGAC se vinculan principalmente con procesos de formación docente y el número de integrantes es de tres o cuatro docentes. Tres CA participan en redes nacionales de investigación.

\section{Tabla 1}

Características de los cuerpos académicos participantes

\begin{tabular}{|c|c|c|c|c|c|}
\hline NO. & $\begin{array}{c}\text { DATO PARA LA } \\
\text { IDENTIFICACIÓN } \\
\text { DEL CA }\end{array}$ & $\begin{array}{l}\text { AÑO DE } \\
\text { REGISTRO } \\
\text { ANTE EL } \\
\text { PRODEP }\end{array}$ & $\begin{array}{l}\text { GRADO DE } \\
\text { CONSOLIDACIÓN }\end{array}$ & LGAC & $\begin{array}{c}\text { NÚMERO } \\
\text { DE } \\
\text { INTEGRANTES }\end{array}$ \\
\hline 1 & TCA-1 & 2011 & En formación. & $\begin{array}{l}\text { Desarrollo de } \\
\text { competencias } \\
\text { docentes. }\end{array}$ & 4 \\
\hline 2 & TCA-2 & 2013 & En consolidación. & $\begin{array}{l}\text { Sistematización } \\
\text { metodológica } \\
\text { de los procesos } \\
\text { de aprendizaje } \\
\text { permanente. }\end{array}$ & 3 \\
\hline 3 & BCA-3 & 2014 & En formación. & $\begin{array}{l}\text { Las competencias } \\
\text { profesionales } \\
\text { de los docentes } \\
\text { de las Escuelas } \\
\text { Normales. }\end{array}$ & 4 \\
\hline 4 & FCA-4 & 2014 & En formación. & $\begin{array}{l}\text { La innovación } \\
\text { educativa para } \\
\text { mejorar el } \\
\text { aprendizaje. }\end{array}$ & 4 \\
\hline 5 & BCA-5 & 2016 & En consolidación. & $\begin{array}{l}\text { Práctica } \\
\text { Profesional } \\
\text { Pedagógica. }\end{array}$ & 3 \\
\hline
\end{tabular}

Nota. Elaboración con base en los datos del PRODEP (GobMx, 2021a). 


\subsection{Participantes}

Los sujetos de estudio fueron cinco docentes integrantes de CA de tres EN del estado de Puebla: cuatro mujeres y un hombre, con edades entre 42 y 65 años. Su trayectoria docente varía entre 15 y 37 años, su experiencia en educación normalista va desde los 15 hasta los 25 años y su antigüedad en el CA, con registro ante el PRODEP, es de cinco a 11 años. Cuatro de ellos atienden programas de licenciatura: una en Educación Preescolar, uno en Educación Primaria, uno en la Licenciatura en Enseñanza y Aprendizaje del Español en Educación Secundaria, y una participa en tres programas, aunque la mayor carga horaria la tiene frente a grupo en la Licenciatura en Educación Inicial. La otra docente se encuentra adscrita a un programa de posgrado, atendiendo estudiantes a nivel de maestría.

Los participantes cultivan LGAC individuales, cuyas temáticas se centran en: tutorías y trayectorias estudiantiles, procesos de literacidad, competencias profesionales de los docentes, innovación para mejorar el aprendizaje y la práctica profesional pedagógica. Las asignaturas que imparten se relacionan con los campos de formación específica y la línea de acercamiento a la práctica escolar (observación y práctica docente, trabajo docente y taller de diseño de propuestas didácticas, y análisis del trabajo docente). Cuatro de ellos son líderes del CA al que pertenecen y una es miembro activo.

\subsection{Técnica de recolección de datos}

El acercamiento se realizó a través de entrevistas semiestructuradas, considerando tres categorías (con sus respectivas subcategorías): colegialidad; producción del conocimiento, y difusión del conocimiento.

En relación con la colegialidad se interrogó acerca de la forma en que se organizan y las actividades que realizan durante sus reuniones, las evidencias de su trabajo, las habilidades con las que cuentan para el trabajo colaborativo, los perfiles de los docentes que permiten la complementariedad, las características de la comunicación, la confianza y el compromiso, así como acerca de la participación en redes académicas, preguntando directamente si pertenecen a alguna y el tipo de participación que tienen, así como el campo y las temáticas desde las cuales generan sus producciones.

Para indagar sobre la producción del conocimiento, se interrogó sobre aspectos metodológicos de la producción que ellos realizan, como: los problemas y principales objetos de investigación que abordan, así como los mecanismos y el proceso que siguen para realizar los proyectos; se cuestionó sobre lo que produce el CA, si esa producción es innovadora y el por qué lo consideran así.

En cuanto a la difusión del conocimiento se interrogó acerca de las personas responsables para realizarla, la dinámica que tienen dentro del CA, el alcance de sus producciones, y el uso de la tecnología de la información y la comunicación (TIC) en los procesos investigativos y de difusión.

\subsection{Técnica de análisis de datos}

La información proporcionada por los participantes fue tratada por medio de un análisis de contenido, técnica que «permite de manera válida, interpretar textos y documentos de forma explícita o implícita» (Díaz, 2018, p. 7). De acuerdo con Bardin (2002, p. 71), las fases de 
organización en esta técnica son tres: «1) El preanálisis, 2) El aprovechamiento del material y 3) El tratamiento de los resultados, la inferencia y la interpretación, cada una con actividades concretas que permiten llegar a la interpretación de los resultados».

\section{Figura 2}

Fases de la organización en el análisis del contenido

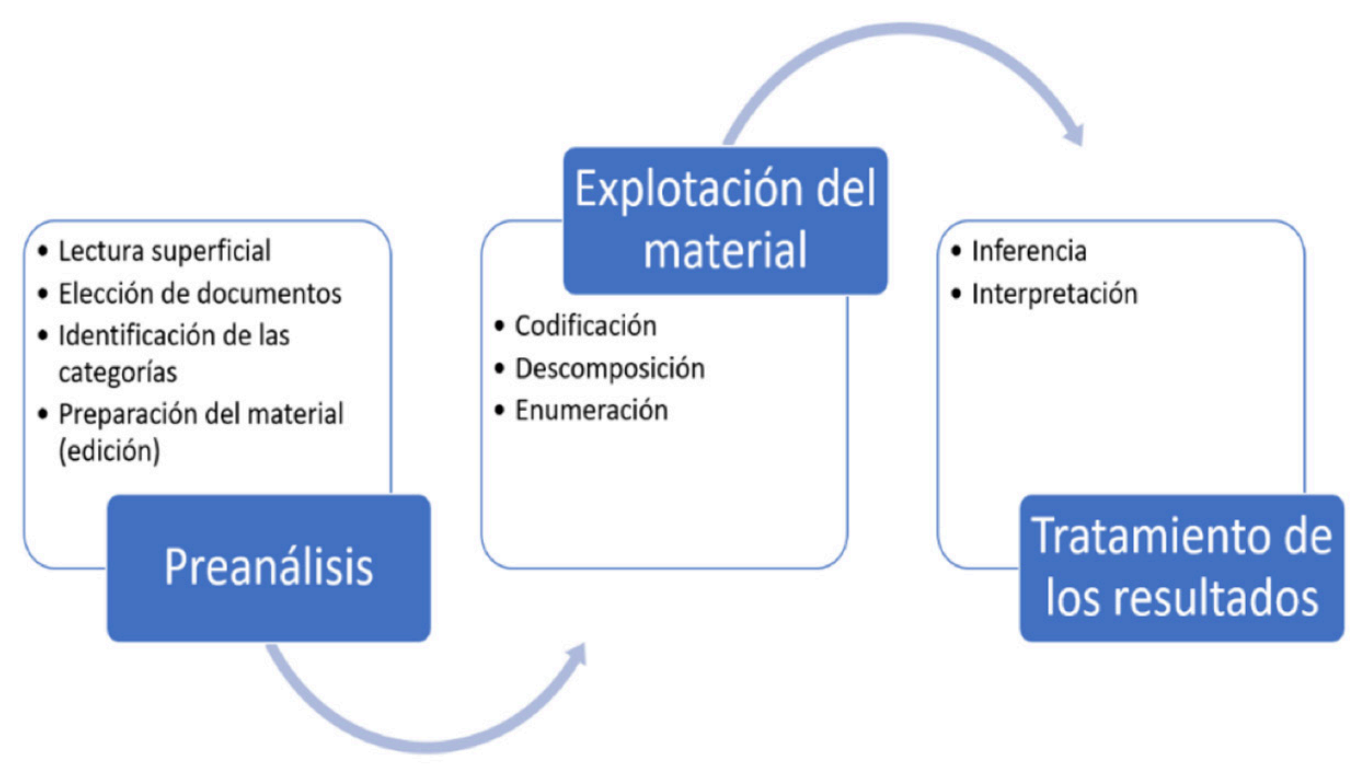

Nota. Elaboración con base en información de Bardin (2002).

\section{RESULTADOS}

En este apartado se describen los hallazgos derivados de las entrevistas a los cinco participantes, a partir de las tres categorías preestablecidas y el objetivo de investigación: colegialidad, producción del conocimiento y difusión del conocimiento.

\subsection{Colegialidad}

A partir de los testimonios de los participantes, la colegialidad en los CA se expresa de varias formas: 
Tabla 2

Testimonios de los participantes en relación con la colegialidad

TEMÁTICAS EXPLORADAS

Tiempo y espacio para la investigación

Frecuencia de las reuniones

\begin{tabular}{|c|}
\hline $\begin{array}{c}\text { Actividades que se } \\
\text { realizan }\end{array}$ \\
\hline
\end{tabular}

Evidencias de trabajo

una

«Tenemos las actas como evidencias, la firma de asistencia, fotografías; tenemos una carpeta donde se van integrando todas las actividades, pero primordialmente nos vamos a la producción, qué es que lo que estamos haciendo, y cómo lo estamos haciendo» (FCA4LM).

Toma de decisiones,
tensiones y conflictos
tensiones y conflictos

«Pues yo creo que es esencial no estar de acuerdo siempre. Yo creo que eso nos hubiera llevado por otro camino. A mí me parece que lo rico de esta comunicación es el respeto, el respeto a la posición [...], a la postura teórica metodológica que se quiere asumir en cierto proyecto y el acompañamiento del otro» (TCA2LM).

\begin{tabular}{|c|l|c|c|c|c|c|}
\hline $\begin{array}{c}\text { Forma en que } \\
\text { dirimen desacuerdos }\end{array}$ & $\begin{array}{l}\text { «[...] como que a veces salen diversos artículos con otros enfoques, pero me parece } \\
\text { que nos permite esto [...] respetar lo que cada uno cree y manifiesta con sus } \\
\text { argumentos» (TCA2LM). }\end{array}$ \\
\hline Trabajo colaborativo & $\begin{array}{l}\text { «De repente hacemos un artículo y es revisado, y a lo mejor lo hacemos solo dos, } \\
\text { y lo revisan los otros dos, entonces de repente uno está leyendo y piensa que ya } \\
\text { está todo limpio, todo bien, y viene la mirada de los otros compañeros, y con ello } \\
\text { se mejora» (FCA4LM). }\end{array}$ \\
\hline Complementariedad & $\begin{array}{l}\text { «[...] pero la líder y una de las colaboradoras son buenísimas de la tecnología, } \\
\text { entonces cada quien pone sus habilidades y cuando le toca a alguien pues lo tiene } \\
\text { que hacer de acuerdo a eso [...]. Uno de los compañeros, es muy bueno en la } \\
\text { redacción, entonces él es un apoyo muy fuerte para nosotros, porque él nos limpia } \\
\text { los documentos» (BCA5IM). }\end{array}$ \\
\hline $\begin{array}{c}\text { Comunicación } \\
\text { A mí me parece que lo rico de esta comunicación es el respeto, el respeto a la } \\
\text { posición [...], postura teórica y metodológica que se quiere asumir en cierto } \\
\text { proyecto y el acompañamiento del otro» (TCA2LM). }\end{array}$ \\
\hline $\begin{array}{c}\text { Confianza y } \\
\text { compromiso }\end{array}$ & $\begin{array}{l}\text { «El equipo lleva varios años conformado y es por medio de la confianza entre } \\
\text { todas que ahí la llevamos» (BCA4IM). }\end{array}$ \\
\hline $\begin{array}{c}\text { Participación en } \\
\text { redes académicas }\end{array}$ & $\begin{array}{l}\text { «Actualmente estamos participando algunos miembros del Cuerpo Académico en } \\
\text { redes, una de ellas una red regional [...]. Pues es [...] de colaboración [...]. También } \\
\text { pertenecemos [...] a una red de colaboración y, sin embargo, la diferencia entre } \\
\text { estas dos redes es que la primera quemencioné es más como de Escuelas Normales } \\
\text { y en la otra ya es con otras instituciones como la BUAP» (TCA1LM). } \\
\text { «El tenor de indagación, de discusión y la investigación y la producción va sobre } \\
\text { tu práctica docente» (BCA3LH). }\end{array}$ \\
\hline
\end{tabular}


Con una organización institucional, en la mayoría de los casos se realizan reuniones frecuentes, facilitadas por un horario en común. El tiempo destinado para ellas es en promedio de una hora y las actividades que realizan incluyen aspectos administrativos, organizativos, de autogestión del conocimiento; los integrantes de los CA comparten artículos y discuten temáticas de interés común, en concordancia con lo que menciona Czarny (2003): que el trabajo colegiado se está constituyendo en un espacio para la discusión académica y la actualización de los profesores.

La forma en que los docentes toman decisiones, realizan acuerdos y dirimen diferencias, se relaciona con el respeto a la opinión del otro; en la mayoría de los casos se menciona una relación de amistad que permite el diálogo franco, con apertura y crítica constructiva. La práctica de trabajo colegiado, como menciona Carro (2017), se significa por la constante comunicación y toma de acuerdos donde los participantes se tratan como iguales, compartiendo responsabilidades y reconocimiento.

Otra de las características que se observa en los CA es el trabajo colaborativo que tiene lugar en la división de tareas de los integrantes, en la ayuda mutua, buscando cumplir con las actividades de los proyectos de investigación e intentando obtener los mejores resultados.

El trabajo colegiado también implica una participación en redes académicas, actividad cubierta en dos CA, participando con otras EN y con universidades. La temática que comparten se vincula con el desarrollo de las competencias docentes, centrada principalmente en la práctica educativa. El trabajo en red es una característica importante para la producción del conocimiento y un requisito indispensable, por parte del PRODEP, para el avance en el grado de consolidación de los CA.

De acuerdo con Maldonado (2010):

[...] trabajar en red, estrechar los lazos de investigación, traspasar las barreras geográficas y participar -de manera virtual o presencial- en grandes colectivos de investigación, es una apuesta que se impone como nueva dinámica de trabajo de los investigadores hoy en día (p. 978).

La misma autora afirma que el trabajo en redes de investigación fomenta la publicación conjunta, permite el acceso a más información y posibilita la actualización continua, entre otras ventajas.

El escenario ideal para el trabajo colegiado, como menciona León (2005), sería concebirlo como una necesidad y como un derecho de todos los docentes, un espacio de indagación, de investigación y acción pedagógica que potencie la necesidad de formación interdisciplinaria, el trabajo en equipo y la construcción de una visión compartida.

\subsection{Producción del conocimiento}

$\mathrm{Al}$ cuestionar a los participantes sobre lo que significa para ellos pertenecer a un CA y realizar investigación educativa, se pueden identificar dos momentos: el inicial, que implica la conformación y el registro del CA, ante el PRODEP; y el momento actual que, con el recorrido realizado, mira a la investigación con ojos diferentes. El primero, percibido como un momento de logro, y el segundo, como una oportunidad de realizar producción de mejor calidad, dejando de lado la subjetividad y el individualismo, adentrándose en el conocimiento, y aportando propuestas de mejora dentro y fuera de la institución.

En el análisis de las entrevistas realizadas, se observan ciertas características en relación con la producción del conocimiento: 
Tabla 3

Testimonios de los participantes en relación con la producción del conocimiento

TEMÁTICAS

ALGUNAS RESPUESTAS DE LOS PARTICIPANTES

EXPLORADAS

Principales

problemas

sobre los que se

investiga

«Los principales problemas, los captamos a partir del acompañamiento que damos a los estudiantes en este trayecto de la práctica profesional; es decir, a partir de la línea de observación y práctica docente [...] los vacíos de conocimiento, son los que nos han dado la pauta para generar a partir de ahí nuestros problemas de investigación» (FCA4LM).

«Percibo que el trabajo del Cuerpo Académico está bien que sea aquí en el aula, pero [...] en cuanto a generación de conocimiento, sí tenemos que generar y tener impacto, y no quedarnos solo en las instituciones, sino generar conocimiento que permita irnos a la comunidad y dar elementos a otras personas, a otras instituciones, irnos a ámbitos más hacia la sociedad» (TCA1LM).

Objetos de

«En ese tenor te conviertes en sujeto y objeto de indagación sobre tu práctica, sobre investigación tu desempeño y entonces la docencia la relacionas con la investigación de manera pertinente hacia la producción del conocimiento y esto, para un servidor, ha representado todo un cambio de la indagación; es decir que la investigación se enfoca en asuntos eminentemente del conocimiento sobre la práctica docente» (BCA3LH).

\begin{tabular}{|c|c|}
\hline $\begin{array}{l}\text { Surgimiento } \\
\text { de las } \\
\text { investigaciones }\end{array}$ & $\begin{array}{l}\text { «Primero, nos ponemos de acuerdo para decir qué es lo que vamos a hacer, y a } \\
\text { partir del proceso que dijimos }[. . .] \text { hacemos un anteproyecto y ese anteproyecto lo } \\
\text { informamos [...]; realmente tenemos una gran ventaja, que es la libertad de poderlo } \\
\text { hacer, porque también hay lugares donde no te dejan hacer nada» (BCA4LM). } \\
\text { «Bueno [...] las investigaciones inician bajo la propuesta de alguno de nosotros } \\
{[\ldots] \text {, creo que así las investigaciones son muy enriquecedoras, porque te van dando }} \\
\text { diferentes caminos, y esos caminos van dando otros, y de repente quieres más y más» } \\
\text { (BCA5IM). } \\
\text { «Las investigaciones se generan principalmente al interior de la escuela, sí, es a partir } \\
\text { de las comisiones, cursos y asignaturas que tenemos» (TCA1LM). }\end{array}$ \\
\hline $\begin{array}{l}\text { Proceso para el } \\
\text { desarrollo de la } \\
\text { investigación }\end{array}$ & $\begin{array}{l}\text { «Tratamos de que las acciones que vamos realizando sean congruentes con el plan, en } \\
\text { el sentido, eh [...] bueno, en que todos los colegas se actualicen en esta metodología de } \\
\text { la indagación de la práctica, bajo los planteamientos epistémicos» (BCA3LH). } \\
\text { «Nos dividimos las tareas: cómo vamos a hacer, qué instrumentos vamos a diseñar, } \\
\text { quién los va a aplicar, cuándo nos vamos a reunir de nuevo y así nos vamos en } \\
\text { ese orden, en ese proceso, hasta poder llegar a la implementación de ese algo que } \\
\text { queríamos producir» (FCA4LM). }\end{array}$ \\
\hline $\begin{array}{l}\text { Tipo de } \\
\text { producción }\end{array}$ & $\begin{array}{l}\text { «[...] en la escuela ya tenemos tres libros institucionales, en donde se refleja el trabajo, } \\
\text { tanto de docentes como de estudiantes o egresados, sobre este tema de la investigación } \\
\text { y la reflexión que hacen de algún tema específico» (TDIR1M). }\end{array}$ \\
\hline $\begin{array}{l}\text { Innovación en la } \\
\text { producción }\end{array}$ & $\begin{array}{l}\text { «La innovación tiene que provocar un cambio ¿no?, un cambio en algo, y entonces en } \\
\text { ese sentido consideramos que hacemos el cambio; por ejemplo, cuando te digo que } \\
\text { empezamos con este manejo de los materiales didácticos innovadores, en este caso de } \\
\text { la caja pitagórica [...] es una innovación, porque lo tenemos a diferencia de otros que } \\
\text { no lo tienen» (FCA4LM). }\end{array}$ \\
\hline
\end{tabular}


Los CA realizan sus investigaciones organizándose desde la planeación de proyectos, se dividen tareas, deciden metodologías, diseñan y aplican instrumentos basándose en los objetivos que se han marcado, todo siguiendo un orden coherente. Lo que producen responde a la normatividad que PRODEP emite para los CA de las EN y varía dependiendo el grado de consolidación, desde elaboración de material didáctico para manejo en el aula, hasta aportaciones en revistas indizadas o diseño de libros, porque los académicos van dirigiendo sus producciones hacia metas más altas.

A partir de los datos recabados y con base en la propuesta de Gibbons et al. (1997), se considera que la producción que realizan los CA se inclina hacia el modo 1, el cual describe «una investigación realizada en la institución, exclusivamente por académicos, unidisciplinar con una estructura jerárquica, cuya validez está dada por una comunidad de pares, que se comunica a través de un evento académico» (Loza et al., 2019, pp. 8-9). Pero algunos profesores son conscientes de que las investigaciones deben transitar hacia el modo 2, en el que, como lo menciona Gaeta (2020), se piensa

en el problema y no en el tema, se vincula a otras disciplinas, y más allá de las instituciones, se piensa en la sociedad; lo que implica estar abiertos al aprendizaje y a lo que aporten otras áreas del conocimiento (p. 77).

Esto podría considerarse obligado, sabiendo que se trata de instituciones formadoras de los futuros docentes del país.

En las investigaciones que realizan los participantes, atienden problemáticas identificadas personalmente o en interior del CA; no se refiere ningún caso de investigaciones que respondan a diagnósticos institucionales o a solicitud de las autoridades. Los objetos de estudio en su mayoría se centran en aspectos áulicos, la metodología es generalmente cualitativa y las aportaciones pretenden la innovación en algún sentido.

\subsection{Difusión del conocimiento}

Los docentes de los CA participan en foros, mesas de discusión con expertos, congresos, mesas redondas, e incluso escriben artículos para revistas especializadas, capítulos de libros o libros, y consideran que la difusión de la producción del conocimiento es responsabilidad de tres agentes: 1 ) del autor o los autores de la investigación; 2) de la autoridad educativa institucional, y 3) de las personas que organizan diversos foros para tal fin.

Algunas de las opiniones de los participantes, vinculadas con la difusión del conocimiento, se expresan en la siguiente tabla: 
Tabla 4

Testimonios de los participantes en relación con la difusión del conocimiento

\begin{tabular}{|c|l|}
$\begin{array}{c}\text { TEMÁTICAS } \\
\text { EXPLORADAS }\end{array}$ & \multicolumn{1}{c|}{ ALGUNAS RESPUESTAS DE LOS PARTICIPANTES } \\
\hline $\begin{array}{c}\text { Dinámica para } \\
\text { la difusión }\end{array}$ & $\begin{array}{l}\text { «Se busca el congreso que alinea los trabajos que nosotros tenemos para poder } \\
\text { llevar una ponencia. Es lo que hemos buscado como proceso de difusión [...]» } \\
\text { (FCA4LM). } \\
\text { «Nos dimos cuenta que los congresos pues, ya no nos estaban aportando lo } \\
\text { que necesitábamos como cuerpo, entonces lo que seguía era empezar a escribir } \\
\text { artículos que se puedan publicar en las revistas; entonces han sido las decisiones } \\
\text { un tanto como lógicas del crecimiento o maduración del cuerpo» (TCA2LM). }\end{array}$ \\
\hline $\begin{array}{c}\text { Alcance de la } \\
\text { difusión }\end{array}$ & $\begin{array}{l}\text { «No ha tenido el alcance que yo quisiera que estos procesos tuvieran, pero eso } \\
\text { no nos hace quedarnos [...], no sabemos hasta dónde ha llegado. Hubo una } \\
\text { participación que tuvimos, un compañero y yo, en un congreso de la BUAP y } \\
\text { de ahí vino gente de otras partes del mundo, investigadores. Ahí pudimos } \\
\text { hacer una cita con una investigadora de Colombia que estaba interesada en } \\
\text { nuestra participación y quería que interactuáramos con ella y con sus alumnos» } \\
\text { (FCA4LM). }\end{array}$ \\
\hline Uso de la TIC & $\begin{array}{l}\text { «La tecnología es determinante: el grabarte, el grabar en una clase, tu discurso } \\
\text { en la entrevista con los mismos chicos, tanto en el aula como fuera de ella, hacer } \\
\text { grupos focales y entrevistarlos, en fin [...] una serie de información que te permita } \\
\text { triangular esta para obtener cierto acercamiento a la teorización es determinante» } \\
\text { (BCA3LH). }\end{array}$ \\
\hline Limitantes & $\begin{array}{l}\text { «Nosotros somos inquietos y buscamos si hay un congreso y, si lo hay y pedimos } \\
\text { permiso, nos dan un oficio de comisión; dinero no nos dan, solo nos dan permiso } \\
\text { y allá a donde vamos saben lo que hicimos» (BCA5IM). }\end{array}$ \\
\hline
\end{tabular}

En los CA de las EN, son los docentes, autores de las investigaciones, los que buscan los espacios para difundir su producción (principalmente congresos cuyas memorias cuenten con la expedición del ISSN). En ocasiones esto se dificulta, porque la asistencia a congresos implica recursos financieros que la mayoría de las veces deben ser solventados por el investigador. Es por ello que actualmente se mira hacia otra posibilidad: las revistas indizadas que, si bien exigen mayor rigor metodológico e implican más tiempo en la gestión, son el recurso ideal para hacer más extensa la difusión de los resultados.

Los docentes están conscientes de que sus producciones tienen un alcance limitado; en algunas ocasiones solo son consideradas dentro de la EN; sin embargo, tienen claro que sus resultados deben impactar en la sociedad y poseen la firme certeza de que su trabajo es importante no solo para ellos y sus estudiantes, sino también para las comunidades en las que se desenvuelven.

La investigación que realizan los CA atiende a un propósito educativo, generando producciones que por el momento impactan directamente en las aulas y en las condiciones institucionales; no obstante, algunas de sus producciones llegan a las escuelas de educación básica, innovando en algunos aspectos y reforzando metodologías en otros. Estas acciones coinciden con la propuesta de Sañudo (2010), quien menciona que la elección de los objetos de investigación y la forma en que se investiga debe generar cambios en las instituciones, 
«esto implica un horizonte aceptado de la innovación viable, un estilo para enfocar los problemas y un conjunto de hábitos y procedimientos aceptados por la comunidad académica para enfrentar las demandas» (pp. 3-4).

\section{CONCLUSIÓN}

Al analizar la contribución de la colegialidad para la producción y difusión del conocimiento en los CA de las EN, del estado de Puebla, se perciben varias aristas.

Inicialmente, una de las principales características para que se conforme un $\mathrm{CA}$, es que los integrantes mantengan entre sí una relación cordial, y tengan objetivos y metas comunes. Este es un primer paso del que surge una colegialidad que da sustento a todos los procesos y las producciones en el grupo de investigación. Establecida la intención, los docentes inician un trabajo colegiado que parte del diseño de un plan de acción, el cual incluye en primera instancia, reuniones frecuentes, en que se gestan proyectos, se intercambian conocimientos, se diseñan instrumentos $y$, entre otros, se atienden aspectos administrativos para cumplir con requerimientos institucionales internos (de la EN) o externos (ante el PRODEP).

En ocasiones, la toma de acuerdos en las reuniones es complicada, pero los docentes la solventan con la práctica de valores -específicamente con respeto y tolerancia ante el argumento disímil-, lo cual también es un aspecto central en el desarrollo del trabajo colegiado. Cabe mencionar que algunas veces el tiempo destinado en la organización institucional para realizar reuniones de trabajo no es suficiente, por lo que los docentes buscan espacios fuera de sus horarios para sus encuentros, que dejan ver su responsabilidad y compromiso para el cumplimiento de sus objetivos y metas.

Los integrantes de los CA han transitado juntos durante cierto tiempo, lo cual ha establecido una cultura colegiada en la que permea un ambiente de camaradería, siendo la comunicación un aspecto medular en los procesos que realizan. Esta característica se enuncia en tres sentidos: al interior del CA, al interior de la EN y con otras instituciones que apoyan o fortalecen las funciones; ello identifica al trabajo colaborativo en redes de investigación, aspecto que interesa a los docentes como parte de un requisito para avanzar en el grado de consolidación, y como parte de un apoyo para mejorar la producción y la difusión que generan.

Las temáticas que abordan se centran principalmente en trabajos dentro de la institución, del aula y quizá, los más aventurados, investigan en escuelas de educación básica en donde los estudiantes normalistas realizan su práctica docente, siendo los aspectos de formación integral y los didácticos, los principales objetos de estudio, lo cual también es consensado en un ambiente colegiado entre los integrantes, al igual que las metodologías y el desarrollo de las investigaciones. Con base en ello, los académicos generan, desde la colegialidad, ciertas innovaciones con sus proyectos, tratando de aportar diferentes formas de abordar las problemáticas, logrando así nuevas ideas para el ejercicio de la práctica docente, con cambios en el aula, en la institución o en las escuelas de educación básica.

De acuerdo con la propuesta de Gibbons et al. (1997), la producción del conocimiento que realizan los CA, se inclina hacia el modo 1, lo cual nuevamente deja ver un aspecto de la colegialidad cimentado en la organización y comunicación entre compañeros, y validado por colegas. Aunque para algunos queda clara la necesidad de transitar hacia otro modo de producir el conocimiento, aún no hay certeza de las condiciones o formas para lograrlo.

La colegialidad para la difusión de la producción del conocimiento presenta una dinámica semejante entre los CA participantes: son los mismos académicos los que buscan las 
opciones para esta tarea. Parten desde la búsqueda de eventos académicos en donde presentan ponencias, hasta la identificación de revistas indizadas, pasando por el diseño de libros, cuyos capítulos generalmente son los resultados de la investigación que realizan. El alcance de su producción también es versátil, llegando en ocasiones a tener una proyección nacional o internacional, pero también existe una coincidencia entre ellos: la producción que realizan se conoce poco en la EN o se desconoce.

Los resultados expresados en la presente investigación, abren la posibilidad de dar seguimiento a nuevos objetos de estudio en torno a los CA de las EN, no solo con un alcance estatal o regional, sino con una mirada más amplia que permita caracterizar a las instituciones formadoras de docentes a nivel nacional. Sería interesante conocer, por ejemplo, acerca del impacto de la producción colegiada que generan, o quizá, escudriñar sobre la constitución y el funcionamiento de redes de investigación entre las EN, lo cual es un tema pendiente, ya que se identifican temas y líneas de investigación comunes, pero no logran concretarse como estrategia de cooperación o de colaboración académica.

En esta investigación se ha podido penetrar en el quehacer cotidiano de los CA de las EN de Puebla, y se han logrado identificar algunas prácticas colegiadas que asumen los integrantes, para la producción y difusión del conocimiento que generan. El acercamiento a las condiciones reales de trabajo dentro de las instituciones, deja ver que los formadores de docentes en estos grupos epistémicos, se esfuerzan por adquirir una cultura diferente que les está llevando a incursionar en procesos investigativos cada vez más sólidos, para el logro de una transformación real del normalismo poblano.

\section{REFERENCIAS}

Antúnez, S. (1999). El trabajo en equipo de los profesores y profesoras: factor de calidad, necesidad y problema. El papel de los directivos escolares. Educar, 24, 88-110.

Asociación Nacional de Universidades e Instituciones de Educación Superior [ANUIES], (2019). Redes de colaboración. En http://www.anuies.mx/anuies/ redes-de-colaboracion

Bakieva, M. (2011). Colegialidad docente. Una evidencia de Validación de Constructo para el diseño de un instrumento de evaluación. Universitat de València.

Bardin, L. (2002). Análisis de contenido. Akai.

Becher, T. (1992). Las disciplinas y el académico. Universidad Futura, 4(10). UAM-Azcapotzalco.

Blumer, H. (1982). El interaccionismo simbólico: perspectiva y método. Hora.

Cano, M. (2010). La investigación colaborativa: Una experiencia en el desarrollo de un proyecto educativo. Ciencia Administrativa, 61-67. https://www.uv.mx/iiesca/ files/2012/12/ colaborativa2010-1.pdf

Carro, A. (2017). El trabajo colegiado en la investigación: El cuerpo académico. Gestión y Políticas Educativas de la Universidad Autónoma de Tlaxcala. [Ponencia]. San Luis Potosí. COMIE.

Chíquiza, M. (2015). La nueva producción del conocimiento y su relación con la educación media. $2^{\circ}$ Simposio Internacional de Postgrados en temas y problemas de 
investigación en educación. Retos y desafios de la educación en la época de la inclusión y la interculturalidad. Universidad de Santo Tomás, Vicerrectoría de Universidad abierta y a distancia, Facultad de educación. https:// documen.site/download/la-nueva-produccion-del-conocimiento-y-su-relacion pdf

Czarny, G. (2003). Las escuelas normales frente al cambio: Un estudio de seguimiento a la aplicación del Plan de Estudio 1997. Cuadernos de discusión, 16. SEP.

Diario Oficial de la Federación [DOF] (2013). ACUERDO número 712 por el que se emiten las Reglas de Operación del Programa para el Desarrollo Profesional Docente. En https: / / sep. gob.mx/work/models/sep1/Resource/ efe3ced7-58c6-476c-874a-56678397baa4/ a712.pdf

Díaz, C. (2018). Investigación cualitativa y análisis de contenido temático. Orientación intelectual de revista Universum. Revista General de Información y Documentación, 28, 119-142. En file:// / C:/Users/lupit/Downloads/60813-Texto\%20del\%20art\%C3\% ADculo-4564456553017-5-10-20180720.pdf

Domínguez-Gaona, M., Crhová, J. y Molina-Landeros, R. (2015). La investigación colaborativa: las creencias de los docentes de lenguas. Revista Iberoamericana de Educación Superior, 6(17), 119-134. https:// doi.org/10.1016/j.rides.2014.09.001

Espinosa, M. E. (2008). El trabajo colegiado en las Escuelas Normales y la evaluación de sus planes de estudio. CPU-e, Revista de Investigación Educativa 7, 1-14 http:/ / www. uv.mx/cpue/num7/opinion/espinosa trabajo_colegiado.htmll

Gaeta, M. (2020). Uso y distribución del conocimiento en investigación educativa. En Reflexiones sobre investigación educativa en la región centro sur desde sus actores, 73-81. Universidad Iberoamericana Puebla.

Gibbons, Limoges, Nowotny, Schwartzman, Scott y Trow. (1997). La nueva producción del conocimiento. La dinámica de la ciencia y la investigación de las sociedades contemporáneas. Pomares-Corredor.

Gobierno de México [GobMx], (2021). Cuerpos académicos reconocidos por PRODEP. Programa para el Desarrollo Profesional Docente, para el Tipo Superior (PRODEP) http:// promep.sep.gob.mx/CA1/index.php?RELOAD $=0$

Hernández, P. (2001). La producción del conocimiento científico como base para determinar perfiles de usuarios. Investigación Bibliotecológica, 15(30), 29-64.

León, A. (2005). El trabajo colegiado en las escuelas de Educación Básica. Una aproximación desde la perspectiva de la gestión y la formación continua de los docentes. EDUCARE, 2.

López, M. (2008). Redes de apoyo para promover la inclusión educativa: Una revisión de algunos equipos y recursos. REICE, 6(2), 200-211.

Loza, M., Merino, C. y Ceja, S. (2019) La producción del conocimiento en las Escuelas Normales de México: una mirada desde el CONISEN [XV Congreso Nacional de Investigación Educativa]. COMIE 2019. Acapulco, Guerrero, México.

Maldonado, E. (2010). Trabajar en redes: nuevo modelo de investigación. Revista mexicana de investigación educativa, 15(46), 975-980. En http://www.scielo.org.mx/scielo. php?script=sci arttext\&pid=S1405-66662010000300014\&lng=es\&tlng=es 
Martínez, G. (2015) La colegialidad es un principio de la organización académica vigente en la práctica cotidiana del CCH. Pero, ¿su operatividad es cotidiana? Nuevos cuadernos el Colegio, UNAM (5), 15-35. http://memoria.cch.unam.mx/tmp/pdf/16/ NCC_No5_ene-mar_2015_1559167686.pdf

Morelli, N. (2000). Trabajo en equipo. En: http:/ / www.marketportal.com.ar/soc/TE/pdf

Rayas, J. (2011). La colegialidad en los procesos de formación permanente de los profesores de educación primaria [XI Congreso Nacional de Investigación Educativa]. COMIE 2011. México, D.F., México.

Ritzer, G. (1997). Teoría sociológica contemporánea. McGraw-Hill.

Rizo, M. (2006). La interacción y la comunicación desde los enfoques de la psicología social y la sociología fenomenológica. Breve exploración teórica. Análisis, 33, 45-62.

Sañudo, L. (2010). La producción y uso del conocimiento educativo [Congreso Iberoamericano de educación, Buenos Aires]. En http:/ / www.adeepra.org.ar/congresos/Congreso\%20IBEROAMERICANO/EIC/R0896 Sanudo.pdf

Sañudo, L (2014). Hacia un modelo de gestión del conocimiento educativo para instituciones de educación superior y centros de investigación [Congreso Iberoamericano de Ciencia, Tecnología, Innovación y Educación, Buenos Aires]. 